\title{
Mangrove vegetation and bird communities around Tegal Port, Central Java, Indonesia
}

\author{
SLAMET ISWORO ${ }^{1, \bullet}$, POERNA SRI OETARI ${ }^{2}$ \\ ${ }^{1}$ Departement of Environmental Health, Universitas Dian Nuswantoro, J1. Imam Bonjol 207, Semarang 50131, Central Java, Indonesia. \\ Tel.: +62-24-3517261, Fax.: +62-24-3569684, `email: slamet.isworo@dsn.dinus.ac.id; slametisworo512@gmail.com \\ ${ }^{2}$ Environmental Impact Assessment. PT. Mitra Adhi Pranata. Jl. Cemara IV No.45, Padangsari, Banyumanik, Semarang 50263, Central Java, Indonesia
}

Manuscript received: 5 November 2019. Revision accepted: 22 March 2020.

\begin{abstract}
Isworo S, Oetari PS. 2020. Mangrove vegetation and bird communities around Tegal Port, Central Java, Indonesia. Biodiversitas 21: 1551-1560. The ecological balance in Tegal harbor area needs to get serious attention due to the impacts of Tegal Port activities. For this purpose, the diversity of mangrove and avifauna (bird) can be used as bio-indicators of the ecological stability of the area. This study aimed to assess and analyze the diversity of plants and avifauna (birds) that live in mangrove and coastal zones around Tegal Port development area. Data collection was conducted using purposive random sampling method on two sites for vegetation and three sites for avifauna. The importance value index, Shannon-Wiener diversity index and Sorensen's similarity index was calculated for the vegetation. For birds, we calculated the diversity and similarity index as well as identified their conservation status based on the IUCN Red List, CITES and government regulations. In terms of vegetation, Rhizophora mucronata and Avicennia marina dominate Site 2 (Tegalsari) while Casuarina equisetifolia and R. mucronata dominate Site 3 (Alam Indah Beach). Overall, the vegetation has low biodiversity index and similarity between sites is also low. For bird, there are 37 species from 18 families. Site 1 (Muara Reja) has the highest number of species with 29 species, followed by Site 2 (Tegalsari) with 25 species, and Site 3 (Alam Indah Beach) with 22 species. The three sites have moderate diversity index and there is high similarity index between sites. All bird species found in the study sites are listed as Least Concern under IUCN Red List, while there are two species protected under national protection law, i.e. Ardea alba and Glareola maldivarum. In general, the condition of mangrove vegetation around Tegal Port has low diversity. This suggests that the pressure on the environment is quite high, causing only few plant species to be able to live and dominate the ecosystem. The limited vegetation also causes birds community, especially the protected A. alba and G. maldivarum in Site 2, becomes increasingly difficult to find, because places to find food, breeding places and places to rest are disturbed.
\end{abstract}

Keywords: Avifauna, birds community, diversity and flatness index, ecological stability, mangrove vegetation

\section{INTRODUCTION}

Seaport development is often required to boost national and regional economies to support logistical transportation and distribution. Nonetheless, the development of seaport causes land-use change, particularly the coastal and mangrove area. Seaport development in marine areas includes the construction of docks, breakwaters, dredging of port ponds, shipping lines and dumping activities, while the development in land area includes the construction of access roads and port facilities.

The development of Tegal Port in Central Java Province, Indonesia is planned to utilize coastal and land areas. While this development is in accordance with national and regional regulations (i.e. Huda 2013; Nasution 2017), this development can certainly cause impacts on surrounding environment, including the flora and fauna community existing around the port development area. Flora and fauna are environmental components that support ecological system, so that safeguarding them is important to consider in the development of Tegal Port. Preserving the existence of various species of flora and fauna living in coastal and mangrove ecosystems around Tegal Port is one strategy to mitigate the impacts of its development (Sukardjo 1993). This strategy of retaining and conserving biodiversity within industrial areas has been increasingly important in industrial sector, as in the case of mining concession (Fiqa et al. 2019), gas refinery (Sudrajat and Putro 2019) and geothermal power plant (Husodo et al. 2020).

Mangroves have special physiological properties in their tissues to adapt with environment with high level of salinity. Mangroves also have adaptations through the root system to support themselves in mud sediments. Mangrove forests in Tegal are communities of mangrove trees that grow along Java Sea coastline and are affected by tides. The condition of mangroves along the coast in Tegal District is very alarming. Most of mangrove forests in Tegal have experienced severe degradation because of conversion into shrimp ponds, exploitation of mangrove trees for firewood and timber as well as coastal abrasion. Currently, efforts to restore mangrove ecosystems in Tegal have been conducted including mangrove areas around Tegal Port, which is conducted by communities and facilitated by the port management. Mangrove ecosystem around Tegal Port is located in the pond area which is no longer used by community. Some species in mangrove and coastal ecosystems in the nearby area of Tegal Port (i.e. Alam Indah Beach) include Casuarina sp., Rhizophora mucronata, Avicennia marina, and Terminalia catappa 
(Ong and Gong 2013), which were planted by communities.

Despite hosting flora community, mangrove ecosystem is also a habitat of various species of avifauna (birds). These birds play an important role in maintaining ecological balance and environmental sustainability especially to control pests and facilitate pollination. In many cases, the existence of birds is often used as bioindicators to assess the state of biodiversity in a region, including in mangroves, because these taxa can occupy a vast habitat, have dynamic flexibility and high mobilization, and have very important roles in the food chain and ecological nets (Gustavsson et al. 2011; Bender et al. 2017). Each bird species has different ability to adapt with environment and can quickly respond to environmental changes, so that changes in environmental conditions can affect behavior and metabolism as a form of adaptive flexibility (Newbold, et al. 2013). In a good condition, mangrove forest is as a suitable habitat for avifauna that can provide them food, shelter and breeding ground.

The mangrove forest area around Tegal Port has diversity of species of birds and vegetation that have not been known specifically and scientifically. Although it is not a conservation forest, it is expected to affect the surrounding environment. Therefore, it is necessary to do research on bird diversity and vegetation in mangrove ecosystem around Tegal Port. This study aimed to assess and analyze the diversity of plants and avifauna (birds) that live in mangroves in the Tegal Port development area.

\section{MATERIALS AND METHODS}

\section{Study period and area}

The fieldwork was carried out from 10 March 2019 to 10 May 2019 in three mangrove areas in Tegal: (1) Muara Reja (109²'25.787"E 6 60'57.057"S), (2) Tegalsari (109 $\left.8^{\prime} 3.58^{\prime \prime E} 6^{\circ} 50^{\prime} 59.259^{\prime \prime S}\right)$, (3) Pantai Alam Indah (109 $\left.8^{\circ} 42.23 " \mathrm{E} \mathrm{6} 6^{\circ} 50^{\prime} 56.929^{\prime \prime S}\right)$ (Figure 1). The average temperature at the study site was $28^{\circ} \mathrm{C}$, the average air pressure ranged from $1009 \mathrm{mb}$ to $1013 \mathrm{mb}$ while air humidity ranged from $77 \%$ to $80 \%$. The general overview of study location is presented in Figure 2.

\section{Data collection \\ Vegetation sampling}

Vegetation sampling was conducted using purposive random sampling method by making 5 observation plots with size of each plot $10 \times 10 \mathrm{~m}$. At observation plot, each individual mangrove was recorded and measured of the diameter at breast height (DBH) and grouped based on strata: trees, poles, and sapling. Category of tree is mangrove with $\mathrm{DBH}>10 \mathrm{~cm}$, while pole $10 \mathrm{~cm}>\mathrm{DBH}>5$ $\mathrm{cm}$ and sapling DBH $<5 \mathrm{~cm}$. Species identification was conducted using guidebooks (e.g. Giesen et al. 2007; Hamzah and Pancawati 2013; Chapman 2016; Hirsch 2016; Tomlinson 2016). Unknown species was labeled with temporary naming, then the samples were collected for further identification.

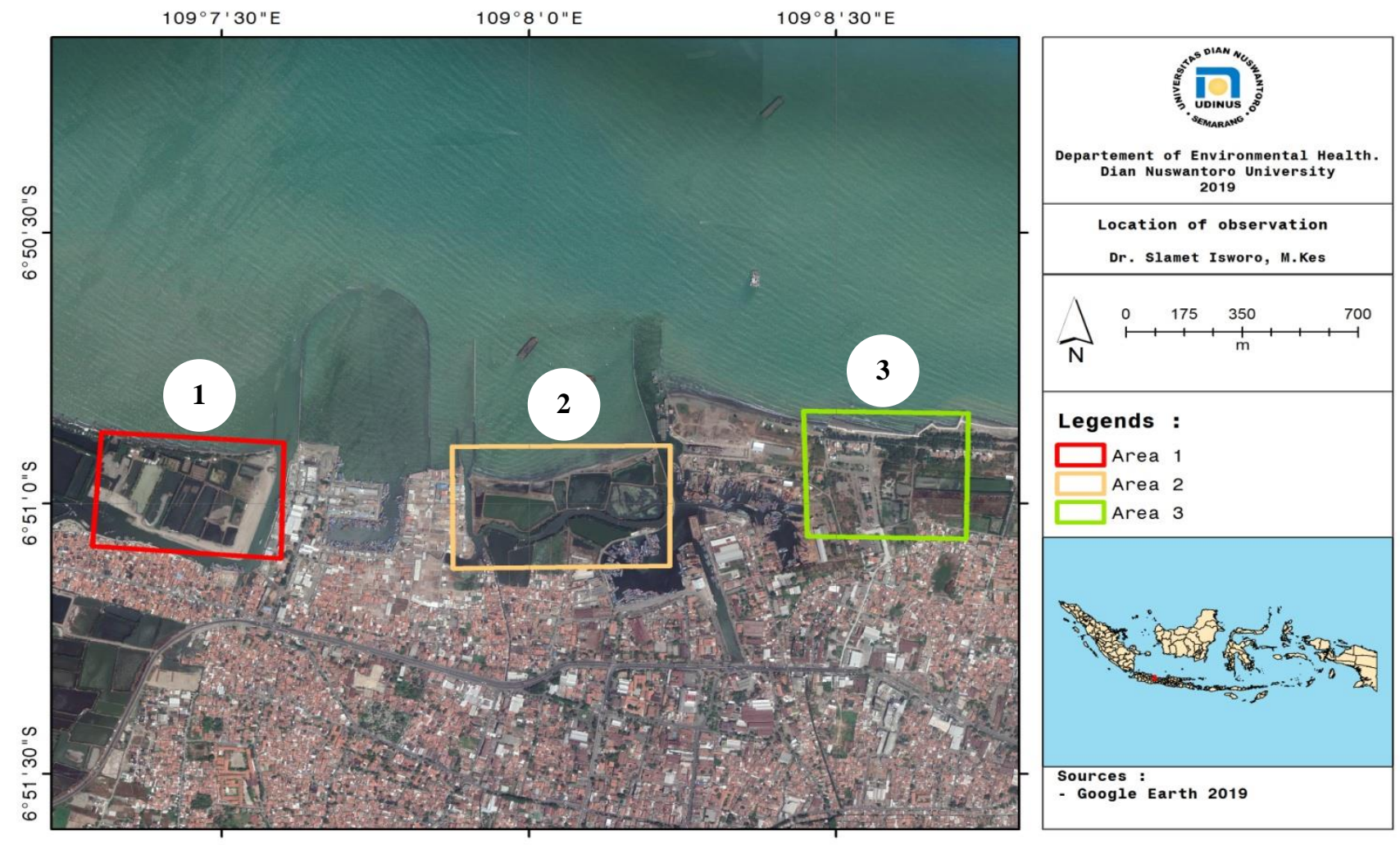

Figure 1. Location of observation in Tegal port, Central Java, Indonesia. A. Muara Reja (area 1), B. Tegal port (area 2), C. Pantai Alam Indah (area 3) 


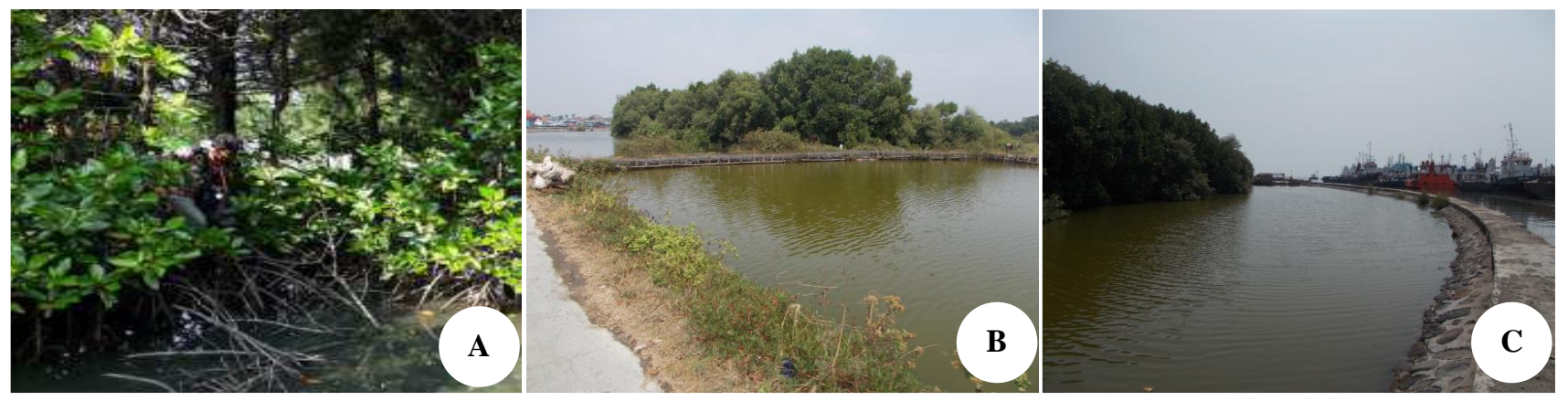

Figure 2. General overview of research sites in Tegal Port, Central Java, Indonesia. A. Muara Reja, B. Tegal port, C. Pantai Alam Indah

\section{Avifauna community}

The diversity of avifauna (birds) was recorded either inside and outside the observation plot. The observed species were identified referring to "A Field Guide Book to Birds of Borneo, Sumatra, Java, and Bali (MacKinnon et al. 2010). Each species was determined its conservation status under the Republic of Indonesia regulation (Noerdjito and Maryanto 2001), Red List of the International Union for Conservation of Nature/IUCN (Beumer and Martens 2013), and Implementation of the Convention on International Trade in Endangered Species of Wild Fauna and Flora/CITES (Raymakers 2006).

\section{Data analysis}

The role of each species was assessed using Important Value Index (IVI). The higher the important value of a species, the greater the contribution of this type to the community (Widyastuti et al. 2018). The calculation of IVI was the sum of relative dominance, relative density and relative frequency as follows (Tauseef et al. 2012).

$$
\mathrm{IV} i=D o R i+D e R i+F R i
$$

IVi : Important value of species i

DoRi: Relative dominance of species $i$

DeRi: Relative density of species i

FRi : Relative Frequency of species $i$ follow:

The relative value for each parameter was calculated as

$$
N R i=\frac{n i}{N}
$$

NR i : Relative species i (DoR, DeR, FR)

$\mathrm{n}$ i : Quantitative value of species i (dominance, density, or frequency)

$\mathrm{N}$ : Total quantitative values of all species

To determine the stability of the mangrove community, a species diversity index was for each growth stage according to the following formula:

$$
H^{\prime}=-\sum P i \cdot \ln P i
$$

$\mathrm{H}^{\prime}$ : Species diversity index

$\mathrm{Pi}$ : Quantitative value of type i/total all quantitative values of all species

The similarity between mangrove communities in the research sites for each growth stage was analyzed using Sorensen index and calculated as follows (Tauseef et al. 2012):

$$
S=\frac{2 W}{a+b} \times 100 \%
$$

$\mathrm{S}$ : Sorensen similarity index

$\mathrm{W}$ : The Lowest number of quantitative values of the species in the two communities compared

a : Quantitative values of all species in the area a

$\mathrm{b}$ : Quantitative values of all species in the area $\mathrm{b}$

For birds, the level of species diversity was analyzed using the Shannon-Wiener diversity index ( $\left.\mathrm{H}^{\prime}\right)$ (Lestari et al. 2017).

\section{RESULTS AND DISCUSSION}

\section{Mangrove community}

For analysis regarding vegetation community, we conducted observation on two sites only, i.e. Site 2 (Tegalsari) and Site 3 (Alam Indah Beach). The results of vegetation analysis in terms of Important Value Index and diversity index are presented in Table 1 for mangroves in Tegalsari (Site 2) and Table 2 for mangroves in Alam Indah Beach (Site 3).

In Tegalsari (Site 2), Rhizophora mucronata has the highest Importance Value Index for tree and pole with value of 161 and 192, respectively while Avicennia marina has the highest for sapling with value of 114 (Table 1). In Alam Indah Beach (Site 3), the most important at tree level is Casuarina equisetifolia IVI of 188, while Rhizophora mucronata has the highest IVI index for pole and sapling with value of 160 and 241, respectively.

The biodiversity is reflected by the Shannon-Wiener Index (H ') (Nyein Aye et al. 2019) and presented in Tables 1 and 2. It can be seen that Tegalsari (Site 2) has a higher diversity index for tree and sapling than Alam Indah Beach (Site 3), but the diversity for pole is lower. 
The similarity between community is not enough to be known merely through the composition of its species and quantitative calculation using Sorensen similarity index has better in reflecting similarity between sites (Maia and Coutinho 2012). Based on Table 3, Tegalsari (Site 2) and Alam Indah Beach (Site 3) has community similarity of $22.73 \%, 63.05 \%$ and $46.4 \%$ for tree, pole, and sapling, respectively.

\section{Avifauna community}

In total, there are 37 bird species across the three sites belong to 17 families (Table 4). Based on observations, it shows that the site with the highest species richness is Site 1 (Muara Reja) with 29 species, followed by Site 2
(Tegalsari) with 25 species, and Site 3 (Alam Indah Beach) with 22 species (Tables 5, 6 and 7).

Observations were also conducted on bird activities for three consecutive days in the observation area (Tables 5, 6 and 7). Daily activities can be grouped into flying, descending, looking for food, looking for food on the ground, perching and making noise.

The diversity index of bird species at each observation site is presented in Figure 3. The results show that Site 1 (Muara Reja) has the highest diversity index compared to the other two locations. This is a sign that Site 1 has a more stable avifauna community than the other sites.

Table 1. Importance value index (IVI) and Diversity Index (H ') of mangroves in Tegalsari (Site 2), Tegal Port, Central Java, Indonesia

\begin{tabular}{|c|c|c|c|c|c|c|c|c|c|c|}
\hline Strata & No & Name & Do & De & Freq & DoR & DeR & FR & IVI & $\mathbf{H}^{\prime}$ \\
\hline \multirow[t]{5}{*}{ Tree } & 1 & Avicennia marina & 197.1 & 0.02 & 0.40 & 32.98 & 27.91 & 22.22 & 83.11 & 0.36 \\
\hline & 2 & Bruguiera cylindrica & 42.4 & 0.00 & 0.20 & 7.09 & 2.33 & 11.11 & 20.52 & 0.18 \\
\hline & 3 & Excoecaria agallocha & 11.5 & 0.00 & 0.20 & 1.92 & 2.33 & 11.11 & 15.35 & 0.15 \\
\hline & 4 & Rhizophora apiculata & 23.9 & 0.00 & 0.20 & 4.00 & 4.65 & 11.11 & 19.76 & 0.18 \\
\hline & 5 & R. mucronata & 322.9 & 0.05 & 0.80 & 54.02 & 62.79 & 44.44 & 161.26 & 0.33 \\
\hline Total & & & 597.8 & 0.09 & 1.80 & 100 & 100 & 100 & 300 & 1.20 \\
\hline \multirow[t]{5}{*}{ Pole } & 1 & Avicennia marina & 70.1 & 0.02 & 0.80 & 12.76 & 14.10 & 33.33 & 60.20 & 0.32 \\
\hline & 2 & Bruguiera cylindrica & 16.6 & 0.01 & 0.40 & 3.02 & 3.85 & 16.67 & 23.53 & 0.20 \\
\hline & 3 & Rhizophora apiculata & 16.9 & 0.00 & 0.20 & 3.07 & 2.56 & 8.33 & 13.97 & 0.14 \\
\hline & 4 & R. mucronata & 440.1 & 0.12 & 0.80 & 80.16 & 78.21 & 33.33 & 191.70 & 0.29 \\
\hline & 5 & Xylocarpus moluccensis & 5.4 & 0.00 & 0.20 & 0.99 & 1.28 & 8.33 & 10.60 & 0.12 \\
\hline Total & & & 549.0 & 0.16 & 2.40 & 100 & 100 & 100 & 300 & 1.00 \\
\hline \multirow[t]{6}{*}{ Sapling } & 1 & Avicennia marina & 194.6 & 0.12 & 0.40 & 50.62 & 50.00 & 13.33 & 113.95 & 0.37 \\
\hline & 2 & Bruguiera cylindrica & 23.2 & 0.02 & 0.80 & 6.05 & 6.78 & 26.67 & 39.49 & 0.27 \\
\hline & 3 & Excoecaria agallocha & 7.3 & 0.00 & 0.40 & 1.91 & 1.69 & 13.33 & 16.93 & 0.16 \\
\hline & 4 & Rhizophora apiculata & 4.1 & 0.00 & 0.20 & 1.08 & 0.85 & 6.67 & 8.59 & 0.10 \\
\hline & 5 & R. mucronata & 146.5 & 0.09 & 0.80 & 38.11 & 37.29 & 26.67 & 102.07 & 0.37 \\
\hline & 6 & R. stylosa & 8.6 & 0.01 & 0.40 & 2.24 & 3.39 & 13.33 & 18.96 & 0.17 \\
\hline Total & & & 384 & 0.24 & 3 & 100 & 100 & 100 & 300 & 1.44 \\
\hline
\end{tabular}

Note: Do: Dominance, De: Density, Freq: Frequency, DoR: Relative dominance, DeR: Relative density, FR: Relative frequency

Table 2. Importance value index (IVI) and Diversity Index (H ') of mangroves in Alam Indah Beach (Site 3), Tegal Port, Central Java, Indonesia

\begin{tabular}{|c|c|c|c|c|c|c|c|c|c|c|}
\hline Strata & No & Name & Do & De & Freq & DoR & DeR & FR & IVI & $\mathbf{H}^{\prime}$ \\
\hline \multirow{5}{*}{ Tree } & 1 & Casuarina equisetifolia & 401.3 & 0.1 & 0.6 & 74.1 & 71.4 & 42.9 & 188.4 & 0.3 \\
\hline & 2 & Hibiscus tiliaceus & 31.2 & 0.0 & 0.2 & 5.8 & 4.8 & 14.3 & 24.8 & 0.2 \\
\hline & 3 & Rhizophora mucronata & 98.4 & 0.0 & 0.4 & 18.2 & 21.4 & 28.6 & 68.2 & 0.3 \\
\hline & 4 & Terminalia catappa & 10.5 & 0.0 & 0.2 & 1.9 & 2.4 & 14.3 & 18.6 & 0.2 \\
\hline & & Total & 541.4 & 0.1 & 1.4 & 100 & 100 & 100 & 300 & 1.0 \\
\hline \multirow[t]{6}{*}{ Pole } & 1 & Avicennia marina & 32.8 & 0.0 & 0.4 & 7.2 & 4.0 & 18.2 & 29.4 & 0.2 \\
\hline & 2 & Casuarina equisetifolia & 61.8 & 0.0 & 0.6 & 13.5 & 6.5 & 27.3 & 47.3 & 0.3 \\
\hline & 3 & Excoecaria agallocha & 101.3 & 0.0 & 0.2 & 22.2 & 12.1 & 9.1 & 43.4 & 0.3 \\
\hline & 4 & Hibiscus tiliaceus & 32.2 & 0.0 & 0.2 & 7.1 & 4.0 & 9.1 & 20.2 & 0.2 \\
\hline & 5 & Rhizophora mucronata & 228.0 & 0.2 & 0.8 & 50.0 & 73.4 & 36.4 & 159.8 & 0.3 \\
\hline & & Total & 456.1 & 0.2 & 2.2 & 100 & 100 & 100 & 300 & 1.3 \\
\hline \multirow[t]{6}{*}{ Sapling } & 1 & Avicennia marina & 43.6 & 0.0 & 0.4 & 3.3 & 3.4 & 20.0 & 26.7 & 0.2 \\
\hline & 2 & Casuarina equisetifolia & 7.3 & 0.0 & 0.2 & 0.5 & 0.5 & 10.0 & 11.0 & 0.1 \\
\hline & 3 & Excoecaria agallocha & 3.8 & 0.0 & 0.2 & 0.3 & 0.2 & 10.0 & 10.5 & 0.1 \\
\hline & 4 & Hibiscus tiliaceus & 3.2 & 0.0 & 0.2 & 0.2 & 0.2 & 10.0 & 10.5 & 0.1 \\
\hline & 5 & Rhizophora mucronata & $1,282.8$ & 0.8 & 1.0 & 95.7 & 95.7 & 50.0 & 241.3 & 0.2 \\
\hline & & Total & $1,340.8$ & 0.9 & 2.0 & 100 & 100 & 100 & 300 & 0.7 \\
\hline
\end{tabular}

Note: Do: Dominance, De: Density, Freq: Frequency, DoR: Relative dominance, DeR: Relative density, FR: Relative frequency 
Tabel 3. Sorensen similarity index between Tegalsari (Site 2) and Alam Indah Beach (Site 3), Tegal Port, Central Java, Indonesia

\begin{tabular}{|c|c|c|c|c|}
\hline \multirow{2}{*}{ Stratum } & \multirow{2}{*}{ Species name } & \multicolumn{2}{|c|}{ Important value index (IVI) } & \multirow{2}{*}{$\mathrm{S}(\%)$} \\
\hline & & Site 2 & Site 3 & \\
\hline \multirow[t]{8}{*}{ Tree } & Avicennia marina & 83.12 & & \\
\hline & Bruguiera cylindrica & 20.52 & & 22.73 \\
\hline & Excoecaria agallocha & 15.35 & & \\
\hline & Rhizophora apiculata & 19.76 & & \\
\hline & Rhizophora mucronata & 161.25 & 68.18 & \\
\hline & Casuarina equisetifolia & & 188.40 & \\
\hline & Hibiscus tiliaceus & & 24.81 & \\
\hline & Terminalia catappa & & 18.61 & \\
\hline Total & & 300 & 300 & \\
\hline \multirow[t]{8}{*}{ Pole } & Avicennia marina & 60.20 & 29.42 & \\
\hline & Casuarina equisetifolia & & 47.27 & \\
\hline & Excoecaria agallocha & & 43.39 & \\
\hline & Hibiscus tiliaceus & & 20.18 & \\
\hline & Rhizophora mucronata & 191.70 & 195,75 & 63.05 \\
\hline & Bruguiera cylindrica & 23.53 & & \\
\hline & Rhizophora apiculata & 13.97 & & \\
\hline & Xylocarpus moluccensis & 10.60 & & \\
\hline Total & & 300 & 300 & \\
\hline \multirow[t]{8}{*}{ Sapling } & Avicennia marina & 113.95 & 26.69 & \\
\hline & Casuarina equisetifolia & & 11.00 & \\
\hline & Excoecaria agallocha & 16.93 & 10.51 & \\
\hline & Hibiscus tiliaceus & & 10.47 & \\
\hline & Rhizophora mucronata & 102.07 & 241.33 & 46.42 \\
\hline & Bruguiera cylindrica & 39.49 & & \\
\hline & Rhizophora apiculata & 8.59 & & \\
\hline & Rhizophora stylosa & 18.96 & & \\
\hline Total & & 300 & 300 & \\
\hline
\end{tabular}

Table 4. Number of individuals and composition of bird species in Tegal Port area, Central Java, Indonesia

\begin{tabular}{lcc}
\hline Family & Percentage & Individual \\
\hline Scolopacidae & 4 & 3 \\
Gareolidae & 4 & 3 \\
Columbidae & 4 & 3 \\
Sternidae & 4 & 3 \\
Cuculidae & 4 & 3 \\
Nectariniidae & 4 & 3 \\
Artamidae & 4 & 3 \\
Chloropsidae & 4 & 3 \\
Pycnonotidae & 5 & 4 \\
Hirundinidae & 5 & 4 \\
Meropidae & 5 & 4 \\
Charadriidae & 5 & 4 \\
Silvidae & 5 & 4 \\
Rallidae & 5 & 4 \\
Apodidae & 8 & 6 \\
Alcedinidae & 11 & 8 \\
Ardeidae & 19 & 14 \\
& 100 & 76 \\
\hline
\end{tabular}

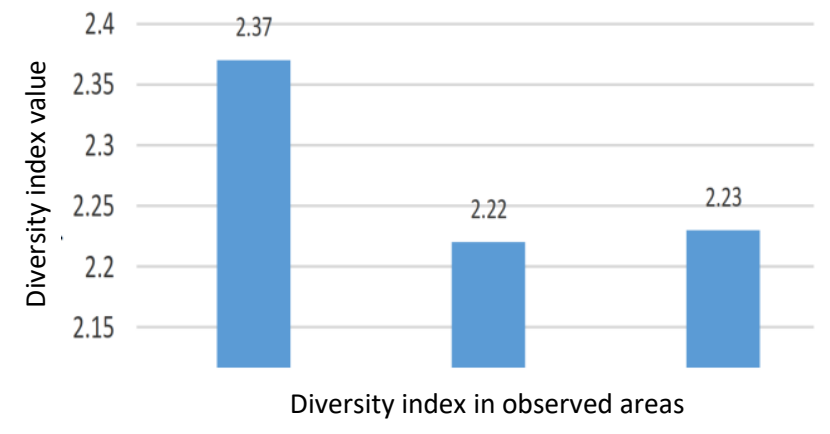

Figure 3. Shannon-Wiener diversity index of bird species at three observation sites in Tegal Port, Central Java, Indonesia

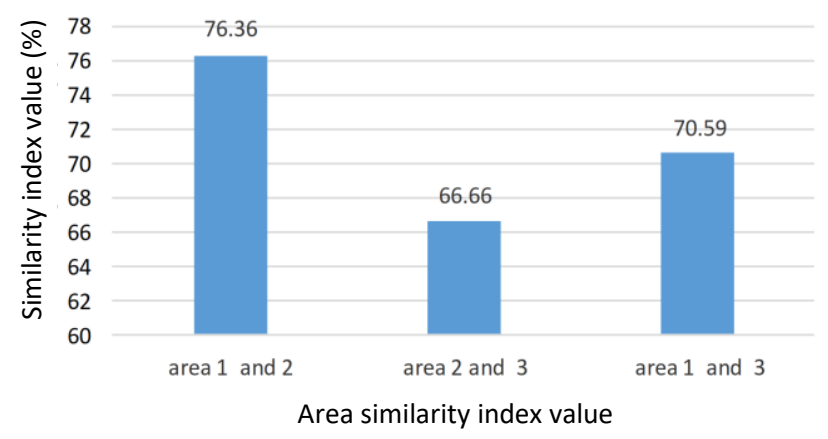

Figure 4. Sorensen's similarity index of bird species between observation sites in Tegal Port, Central Java, Indonesia 
Table 5. Bird's activities observed at Site 1 (Muara Reja), Tegal Port, Central Java, Indonesia

\begin{tabular}{|c|c|c|c|}
\hline Local name & Family & Scientific name & Observed activity \\
\hline Bambangan Kuning & Ardeidae & Ixobrychus sinensis & Fly, find food \\
\hline Blekok Sawah & Ardeidae & Ardeola speciosa & Fly, find food \\
\hline Bondol Haji & Ploceidae & Lonchura maja & Fly, find food \\
\hline Bondol Peking & Ploceidae & Lonchura punctulata & Fly, find food, nest \\
\hline Burung Gereja Erasia & Ploceidae & Passer montanus Lonchura & Fly, perch, find food \\
\hline Burung Madu Sriganti & Nectariniidae & Nectarinia jugularis & Fly, perch, find food \\
\hline Cangak Abu & Ardeidae & Ardea cinerea & Fly \\
\hline Cekakak Australia & Alcedinidae & Todirhamphus sanctus & Fly, perch, find food \\
\hline Cekakak Sungai & Alcedinidae & Todirhamphus chloris & Fly, perch, find food \\
\hline Cerek Kalung Kecil & Charadriidae & Charadrius dubius & Look for food \\
\hline Cerek Tilil & Charadriidae & Charadrius alexandrinus & Look for food \\
\hline Cici Padi & Silvidae & Cisticola juncidis & Fly, perch, find food \\
\hline Cipoh Kacat & Chloropsidae & Aegithina tiphia & Roost, find food \\
\hline Cucak Kutilang & Pycnonotidae & Pycnonotus aurigaster & Fly, perch, find food \\
\hline Dara Laut Sayap Putih & Sternidae & Chlidonias leucopterus & Fly, find food \\
\hline Kareo Padi & Rallidae & Amaurornis phoenicurus & In the bush looking for food \\
\hline Kapinis Rumah & Apodidae & Apus pacificus & Fly \\
\hline Kirik-Kirik Laut & Meropidae & Merops philippinus & Fly \\
\hline Kokokan Laut & Ardeidae & Butorides striatus & Fly, perch, find food \\
\hline Kuntul Kecil & Ardeidae & Egretta garzetta & Fly, perch, find food \\
\hline Layang-Layang Batu & Hirundinidae & Hirundo tahitica & Fly, find food on the ground \\
\hline Layang-Layang Loreng & Hirundinidae & Hirundo striolata & Fly \\
\hline Merbah Cerukcuk & Pycnonotidae & Pycnonotus goiavier & Fly, perch, find food \\
\hline Raja Udang Biru & Alcedinidae & Alcedo coerulescens & Fly, perch, find food \\
\hline Remetuk Laut & Silvidae & Gerygone sulphurea & Noiseless flying \\
\hline Tekukur bIasa & Columbidae & Streptopelia chinensis & Fly, perch, on the ground foraging \\
\hline Trinil Pantai & Scolopacidae & Tringa hypoleucos & looking for food on the beach \\
\hline Walet Linchi & Apodidae & Collocalia linchi & Fly \\
\hline Walet Sarang Putih & Apodidae & Collocalia fusiphaga & Fly \\
\hline
\end{tabular}

Table 6. Bird's activities observed at Site 2 (Tegalsari), Tegal Port, Central Java, Indonesia

\begin{tabular}{llll}
\hline Local name & Family & Scientific name & Observed activity \\
\hline Blekok sawah & Ardeidae & Ardeola speciosa & Fly, find food \\
Bondol haji & Ploceidae & Lonchura maja & Fly, find food \\
Bondol haji & Ploceidae & Lonchura maja & Fly, find food \\
Bondol peking & Ploceidae & Lonchura punctulata & Fly, find food, nest \\
Burung gereja erasia & Ploceidae & Passer montanus & Fly, perch, find food \\
Burung madu sriganti & Nectariniidae & Nectarinia jugularis & Fly, perch, find food \\
Cangak besar & Ardeidae & Ardea alba & Fly, perch, find food \\
Cekakak australia & Alcedinidae & Todirhamphus sanctus & Fly, perch, find food \\
Cekakak sungai & Alcedinidae & Todirhamphus chloris & Fly, perch, find food \\
Cerek kalung kecil & Charadriidae & Charadrius dubius & Look for food \\
Cici padi & Silvidae & Cisticola juncidis & Fly, perch, find food \\
Cucak kutilang & Pycnonotidae & Pycnonotus aurigaster & Fly, perch, find food \\
Kareo padi & Rallidae & Amaurornis phoenicurus & Fly \\
Kokokan laut & Ardeidae & Butorides striatus & Fly \\
Kowak-malam abu & Ardeidae & Nycticorax nycticorax & Fly \\
Kuntul kecil & Ardeidae & Egretta garzetta & Fly, find food \\
Layang-layang batu & Hirundinidae & Hirundo tahitica & Fly, find food on the ground \\
Merbah cerukcuk & Pycnonotidae & Pycnonotus goiavier & Fly, find food on the ground \\
Raja udang biru & Alcedinidae & Alcedo coerulescens & Fly, perch, find food \\
Remetuk laut & Silvidae & Gerygone sulphurea & Noiseless flying \\
Tekukur biasa & Columbidae & Streptopelia chinensis & Fly, perch, on the ground foraging \\
Terik asia & Gareolidae & Glareola maldivarum & Noiseless flying \\
Bambangan kuning & Ardeidae & Ixobrychus sinensis & Fly, perch, find food \\
Tikusan merah & Rallidae & Porzana fusca & looking for food on the beach \\
Walet linchi & Apodidae & Collocalia linchi & Fly \\
\hline
\end{tabular}


Table 7. Bird's activities observed at Site 3 (Alam Indah Beach), Tegal Port, Central Java, Indonesia

\begin{tabular}{llll}
\hline Local name & Family & Scientific name & Observed activity \\
\hline Bambangan Kuning & Scolopacidae & Tringa hypoleucos & Fly, find food \\
Bondol Haji & Ploceidae & Lonchura maja & Fly, find food \\
Bondol Haji & Ploceidae & Lonchura maja & Fly, find food \\
Bondol Peking & Ploceidae & Lonchura punctulata & Fly, find food, nest \\
Burung Gereja Erasia & Ploceidae & Passer montanus & Fly, perch, find food \\
Cekakak Australia & Alcedinidae & Todirhamphus sanctus & Fly, perch, find food \\
Cekakak Sungai & Alcedinidae & Todirhamphus chloris & Fly, perch, find food \\
Cici Padi & Silvidae & Cisticola juncidis & Fly, perch, find food \\
Cucak Kutilang & Pycnonotidae & Pycnonotus aurigaster & Fly, perch, find food \\
Kekep Babi & Artamidae & Artamus leucorhynchus & Fly, perch, find food \\
Kokokan Laut & Ardeidae & Butorides striatus & Fly \\
Kuntul Kecil & Ardeidae & Egretta garzetta & Fly, perch, find food \\
Layang-Layang Batu & Hirundinidae & Hirundo tahitica & Fly \\
Loreng & Hirundinidae & Hirundo striolata & Fly \\
Merbah Cerukcuk & Pycnonotidae & Pycnonotus goiavier & Fly, find food on the ground \\
Raja Udang Biru & Alcedinidae & Alcedo coerulescens & Fly, perch, find food \\
Meninting & Alcedinidae & Alcedo meninting & Fly, perch, find food \\
Remetuk Laut & Silvidae & Gerygone sulphurea & Noiseless flying \\
Tekukur bIasa & Columbidae & Streptopelia chinensis & Fly, perch, foraging \\
Trinil Pantai & Scolopacidae & Tringa hypoleucos & Fly, perch, find food \\
Walet Linchi & Apodidae & Collocalia linchi & Fly \\
Wiwik Uncuing & Cuculidae & Cuculus spulcralis & Noiseless flying \\
\hline
\end{tabular}

Table 8. The conversion status of bird species according to three references

\begin{tabular}{|c|c|c|c|c|c|c|}
\hline \multirow{2}{*}{ Familia } & \multirow{2}{*}{ Indonesian name } & \multirow{2}{*}{ Scientific name } & \multicolumn{3}{|c|}{ Conservation status } & \multirow{2}{*}{ Population trend *: } \\
\hline & & & $(*)$ & $(* *)$ & $(* * *)$ & \\
\hline Ardeidae & Kuntul kecil & Egretta garzetta & not protected & LC & - & Increasing \\
\hline Ardeidae & Cangak Abu & Ardea cinerea & not protected & $\mathrm{LC}$ & - & Unknown \\
\hline Ardeidae & Cangak besar & Ardea alba & protected & $\mathrm{LC}$ & - & Unknown \\
\hline Ardeidae & Kokokan laut & Butorides striatus & not protected & $\mathrm{LC}$ & - & Decreasing \\
\hline Ardeidae & Blekok sawah & Ardeola speciosa & not protected & $\mathrm{LC}$ & - & Unknown \\
\hline Ardeidae & Kowak-malam abu & Nycticorax nycticorax & not protected & $\mathrm{LC}$ & - & Decreasing \\
\hline Ardeidae & Bambangan kuning & Ixobrychus sinensis & not protected & $\mathrm{LC}$ & - & Unknown \\
\hline Rallidae & Tikusan merah & Porzana fusca & not protected & $\mathrm{LC}$ & - & Decreasing \\
\hline Rallidae & Kareo padi & Amaurornis phoenicurus & not protected & $\mathrm{LC}$ & - & Unknown \\
\hline Charadriidae & Cerek-kalung kecil & Charadrius dubius & not protected & $\mathrm{LC}$ & - & Stable \\
\hline Charadriidae & Cerek tilil & Charadrius alexandrinus & not protected & $\mathrm{LC}$ & - & Decreasing \\
\hline Scolopacidae & Trinil pantai & Tringa hypoleucos & not protected & $\mathrm{LC}$ & - & Decreasing \\
\hline Gareolidae & Terik asia & Glareola maldivarum & protected & $\mathrm{LC}$ & - & Decreasing \\
\hline Sternidae & Dara-laut sayap-putih & Chlidonias leucopterus & not protected & $\mathrm{LC}$ & - & Stable \\
\hline Columbidae & Tekukur biasa & Streptopelia chinensis & not protected & $\mathrm{LC}$ & - & Increasing \\
\hline Cuculidae & Wiwik uncuing & Cuculus spulcralis & not protected & $\mathrm{LC}$ & - & Stable \\
\hline Apodidae & Walet linci & Collocalia linchi & not protected & $\mathrm{LC}$ & - & Decreasing \\
\hline Apodidae & Kapinis rumah & Apus pacificus & not protected & $\mathrm{LC}$ & - & Increasing \\
\hline Apodidae & Walet sarang putih & Collocalia fusiphaga & not protected & $\mathrm{LC}$ & - & Decreasing \\
\hline Alcedinidae & Meninting & Alcedo meninting & not protected & $\mathrm{LC}$ & - & Decreasing \\
\hline Alcedinidae & Raja-udang biru & Alcedo coerulescens & not protected & $\mathrm{LC}$ & - & Stable \\
\hline Alcedinidae & Cekakak sungai & Todirhamphus chloris & not protected & $\mathrm{LC}$ & - & Decreasing \\
\hline Alcedinidae & Cekakak australia & Todirhamphus sanctus & not protected & $\mathrm{LC}$ & - & Increasing \\
\hline Meropidae & Kirik-kirik laut & Merops philippinus & not protected & $\mathrm{LC}$ & - & Stable \\
\hline Hirundinidae & Layang-layang batu & Hirundo tahitica & not protected & $\mathrm{LC}$ & - & Unknown \\
\hline Hirundinidae & Layang-layang loreng & Hirundo striolata & not protected & $\mathrm{LC}$ & - & Stable \\
\hline Pycnonotidae & Cucak kutilang & Pycnonotus aurigaster & not protected & $\mathrm{LC}$ & - & Decreasing \\
\hline Pycnonotidae & Merbah cerukcuk & Pycnonotus goiavier & not protected & $\mathrm{LC}$ & - & Increasing \\
\hline Chloropsidae & Cipoh Kacat & Aegithina tiphia & not protected & $\mathrm{LC}$ & - & Unknown \\
\hline Silvidae & Remetuk laut & Gerygone sulphurea & not protected & $\mathrm{LC}$ & - & Decreasing \\
\hline Silvidae & Cici padi & Cisticola juncidis & not protected & $\mathrm{LC}$ & - & Increasing \\
\hline Artamidae & Kekep Babi & Artamus leucorhynchus & not protected & $\mathrm{LC}$ & - & Stable \\
\hline Nectariniidae & Burung-madu sriganti & Nectarinia jugularis & not protected & $\mathrm{LC}$ & - & Stable \\
\hline Ploceidae & Burung-gereja Erasia & Passer montanus Lonchura & not protected & $\mathrm{LC}$ & - & Decreasing \\
\hline Ploceidae & Bondol Jawa & leucogastroides & not protected & $\mathrm{LC}$ & - & Stable \\
\hline Ploceidae & Bondol peking & Lonchura punctulata & not protected & $\mathrm{LC}$ & - & Stable \\
\hline Ploceidae & Bondol haji & Lonchura maja & not protected & $\mathrm{LC}$ & - & Stable \\
\hline
\end{tabular}

Note: (*) Indonesian animal protection law (Lubis 2017) (Reeve 2014); (**) IUCN (IUCN 2014) (Beumer and Martens 2013), (***) CITES (Corella 2012); LC = Least Concern. 
The similarity of bird communities between sites was analyzed using Sorensen's index (Morelli 2013) and is presented in Figure 4. Communities are said to be the same if Sorensen's similarity value reaches more than $60 \%$. From the calculation, it shows that two areas having the most similarity in bird species composition are observed at Site 1 and Site 2.

In this study, the conservation status of bird species was identified referring to Indonesian government regulations, IUCN (The International Union for Conservation of Nature) and CITES (Convention on International Trade in Endangered Species). The list is presented in Table 8.

\section{Discussion}

\section{Mangrove community}

In general, the vegetation in Site 2 (Tegalsari) can be categorized as a mangrove forest with Rhizophora mucronata and Avicennia marina as the main constituent. On the other hand, Site 3 (Alam Indah Beach) can be categorized as coastal forest with the Cypress Sea (Casuarina equisetifolia) dominated the area along with species of shrubs in sandy areas. This is justified by important value index (IVI) and diversity index of such species. The IVI describes the role of a plant species in a vegetation community in which the greater the importance value index of a plant species, the greater the role of the species in the community. The important value index can also be said as a quantitative parameter to express the level of dominance of a species in a plant community. The dominant species in a plant community will have a high importance value index, such the most dominant species will have the highest importance value index (Tauseef et al. 2012; Daly et al. 2018).

Each species has an important role in mangrove community, especially at the growth stages of tree and pole (Giesen et al. 2007). It is justified with the fact that at the time of observation, birds used these two growth stages to support their lives, especially for making nests. In Site 2 (Tegalsari), Rhizophora mucronata has the highest important value index at tree and pole stages, while at sapling category Avicennia marina has the highest index. On the other hand, the tree stage in Site 3 (Alam Indah Beach) is dominated by Cypress Sea (Casuarina equisetifolia) while Rhizophora mucronata dominates the juvenile stages. This is understandable since Site 3 (Alam Indah Beach) is a tourist area. However, with the dominance of Rhizophora mucronata at juvenile stages, it is likely that the vegetation community in this site will be dominated by this species in the future.

Rhizophora mucronata is commonly found to have high density, high frequency and high dominance in mangrove vegetation, indicating that this species has important role in mangrove community and affects the stability of the ecosystem (Lacerda et al. 2017). This also applies to Avicennia marina. Species from the genus of Rhizophora and Avicennia generally dominate Indonesian coastal region, showing a strong mutualism between the two families (Sandilyan and Kathiresan 2012). Rhizophora mucronata is easy to grow either in areas with high or low tidal inundation and it is highly halophytic or salt tolerant. Avicennia marina has high survival ability in which when damaged it can immediately recovering and grow back. Both species also have dense and intertwined breathing roots which are very effective to capture and hold mud. This is an ideal microhabitat for various species of mangrove crabs, snails and fish, resulting in a symbiotic mutualism between organisms (Singh and Odaki 2004). Besides marine fauna, we also observed bird species from the Ardeidae family nesting on Rhizophora mucronata in our study sites. Whereas Avicennia marina at the sapling level becomes nesting place of Leucogastroides sp., Lonchura punctulata, and Lonchura maja.

In term of vegetation similarity, Site 2 and Site 3 has $22.7 \%$ of Sorensen's similarity index for tree stage, while for pole and sapling, they have similarity of $63 \%$ and $46.4 \%$, respectively. The low similarity is likely caused by the difference in vegetation type in which Site 2 tends to be an area with mangrove forest type, while Site 3 tends to show the type of coastal forest. The community similarity index illustrates the level of structural similarity and species composition of the stands being compared. The similarity index value ranges from $0-100 \%$, where the higher the value of the index of species similarity indicates the higher the level of similarity of species between the two communities compared, can also be interpreted that the higher the index value of the similarity of species (Rani et al. 2018).

The results of vegetation analysis show the current ecological conditions of mangrove vegetation around Tegal Port so that the impacts caused by the port activities can be carried out without damaging the surrounding environment and being sustainable (Tomlinson 2016). In general, the condition of mangrove vegetation around Tegal Port has low diversity. This suggests that the pressure on the environment is quite high, causing only few plant species to be able to live and dominate the ecosystem (Kordas, Dumbrell and Woodward 2016).

\section{Avifauna community}

Based on the results, the three study sites consist of 37 species of avifauna (birds) from 18 families. Site 1 (Muara Reja) has the highest number of species with 29 species, followed by Site 2 (Tegalsari) with 25 species, and Site 3 (Alam Indah Beach) with 22 species. Ardeidae is the family with the highest number of species with 7 (19\%) of the total species. This shows that mangrove habitat around Tegal Port is suitable for the Ardeidae family. This family contains protected species, i.e. Ardea alba and Glareola maldivarum (Handayani et al. 2018), both are found in Site 2 . Site 3 has the lowest number of species since it has coastal forest vegetation with a dominance of Lonchura punctulata and Passer montanus.

Based on calculations using the Shannon-Wiener diversity index, it is known that Site 1 has the highest diversity. This shows that Site 1 has better avifauna community stability than the other two sites. This is because, in Site 1, there are no species that are very dominating. Shannon-Wiener diversity index is strongly 
influenced by species abundance, meaning that if there are one species tends to dominate, it can reduce the diversity index overall (Stirling and Wilsey 2001). In addition, the higher diversity index in Site 1 is also due to the high species richness compared to the other two sites since the value of diversity is directly proportional to species richness.

The Sorensen's similarity index for avifauna in the studied area is $76 \%$ between Site 1 and Site 2, 70\% between Site 1 and Site 3, and 66\% between Site 2 and Site 3 . Based on this result, bird communities among the three sites are said to be identified as the Sorensen's similarity value reaches more than $60 \%$ with the highest percentage of similarity is between Site 1 and Site 2. The similarity index is used to determine the composition that underlies the presence or absence of a relationship between the number of species that are common in the two community areas and the total species. The result of this study suggests that the bird population living Tegal Port area can move across the three sites. Birds can move from a site to other sites if the site is no longer able to support them, so they will find suitable areas for their lives. The greater similarity index between regions, the more suitable and the higher the chance of a species to move to the area (Hoda et al. 2017).

All bird species found in the study sites are listed as Least Concern under IUCN Red List, while there are two species protected under national protection law, i.e. A. alba and G. maldivarum (Terik Asia). Based on the Asian Waterbird Census 2008-2015, there are 98 species of settlers and wanderers with 112 thousand individuals observed in Indonesia. Of them, 80 species are categorized as low risk (Least Concern), 11 species approaching endangered (Near Threatened), 2 species are under Vulnerable and 5 species are under Endangered. In 2017, it is predicted that there will be an increase of 142 locations with a total of 103 settlers and wanderers waterbirds monitored, including G. maldivarum and A. alba. This situation is due to disturbance in the habitat of both birds due to anthropogenic activity (Mundkur, Langendoen and Watkins 2017). A. alba is waterbird species that has the behavior of flying more often alone or in small groups, tends to be solitary and unusual in the presence of humans (Green et al. 2016). G. maldivarum is a species of bird from the Glareolidae family, living in groups, flying to catch insects in the air, resting on the ground, foraging for food mingling with other scaffolds, but separated by flight. This bird is a type of insectivorous and has habitats in swamps and rice fields, spread to a height of $500 \mathrm{~m}$ above sea level (Lestari et al. 2017).

In summary, the impact of the development of Tegal Port causes the reduction of mangrove vegetation, so that the avifauna (birds) community, especially $A$. alba and $G$. maldivarum in Site 2 becomes increasingly difficult to find, because places to find food, breeding places and places to rest are disturbed, they will leave their habitat if there is an ecological imbalance in their habitat.

\section{ACKNOWLEDGEMENTS}

This research is a follow-up study of the analysis of environmental impacts in PT. Pelabuhan Indonesia III which manages Tegal Port. The author thanks to the management of PT. PELINDO III Central Java for funding the study and the Mitra Pranata Consultant Company. The author declares that there is no competing interest and this research is in accordance with code of ethics.

\section{REFERENCES}

Bender IMA, Kissling WD, Böhning-Gaese K, Hensen I, Kühn I, Wiegand T, Dehling DM, Schleuning M. 2017. Functionally specialised birds respond flexibly to seasonal changes in fruit availability. J Anim Ecol 86 (4): 800-811.

Beumer C, Martens P. 2013. IUCN and perspectives on biodiversity conservation in a changing world. Biodiv Conserv 22 (13-14): 31053120 .

Chapman VJ. 2016. Coastal Vegetation. Elsevier, New York.

Corella IP. 2012. The need for a better understanding of context when applying CITES regulations: the case of an Indonesian parrotTanimbar corella'. The Trade in Wildlife: Regulation for Conservation. Earthscan: 153

Daly AJ, Baetens JM, De Baets B. 2018. Ecological diversity: measuring the unmeasurable, Mathematics. Multidisciplinary Digital Publishing Institute 6 (7): 119.

Fattorini S, Rigal F, Cardoso P, Borges PAV. 2016. Using species abundance distribution models and diversity indices for biogeographical analyses, Acta Oecologica 70: 21-28.

Fiqa AP, Fauziah F, Lestari DA, Budiharta S. 2019. The importance of insitu conservation area in mining concession in preserving diversity, threatened and potential floras in East Kalimantan, Indonesia. Biodiversitas 20: 198-210.

Giesen W, Wulffraat S, Zieren M, Scholten L. 2007. Mangrove Guidebook for Southeast Asia. FAO Regional Office for Asia and the Pacific, Bangkok.

Green AJ, Soons M, Brochet al., Kleyheeg E. 2016. Dispersal of plants by waterbirds. In: Sekercioglu CH, Wenny DG, Whelan CJ (eds.). Why Birds Matter: Avian Ecological Function and Ecosystem Services. University of Chicago Press Chicago, IL.

Gustavsson J, Cederberg C, Sonesson U. 2011. Global Food Losses and Food Waste. FAO, Rome.

Handayani CNN, Estradivari, Daniel D, Hadian O, Faisal KF, Suciptoa D, Maulida P. 2018. Identification of conservation priority location in Indonesia based on land-sea connectivity. J Coast Ocean 1 (2): 13-26.

Hirsch P. 2016. Routledge Handbook of the Environment in Southeast Asia. Taylor \& Francis, London.

Hoda A, Sapsuha Y, Syafie Y. 2017. The Diversity Of Bird Species In Ternate Island, Biotika. Federal Service for Supervision of Communications and Mass Media of Russian 4 (17): 72-81.

Huda FN. 2013. Implementation of Regional Regulation No. 4 of 2012 concerning Tegal city regional spatial planning (Study of provision of green open space). Journal of Politic and Government Studies 2 (2): 311-320.

Husodo T, Mochtan KP, Shanida SS, Aminuddin SF, Wulandari I, Putra IS, Megantara EN. 2020. Avian diversity in geothermal power plant areas: Case studies in Kamojang, Darajat, and Gunung Salak, West Java, Indonesia. Biodiversitas 21 (3): 1049-1059.

IUCN. 2014. IUCN Red List for birds'. BirdLife International Cambridge, UK

Kordas R, Dumbrell A, Woodward G. 2016. Large-scale ecology: model systems to global perspectives. Academic Press, New York.

Lacerda L.D, Conde J.E, Alarcon C, Alvarez-León R, Bacon R.S, D'Croz L, Kjerfve B, Polaina J, Vannucci M. 2017. Mangrove ecosystems of Latin America and the Caribbean: a summary. ITTO/ISME, Japan.

Lestari YI, Edi W, Alivvy A, Ibadah AS, Sari FY, Nuraini F, Yanuar A, Satriyono A, Riany CF, Saptarini D, Muzaki FK. 2017. Revegetation increase bird diversity in coastal area of Socorejo, Tuban, East JavaIndonesia. AIP Conf Proc 1854: 020023-1-020023-6. DOI: $10.1063 / 1.4985414$ 
Lubis MI. 2017. Implementation of the Convention on International Trade in Endangered Species of Wild Fauna and Flora (CITES) and Law No, 5 of 1990 on the Conservation of Biological Natural Resources and Its Ecosystems in Law Enforcement Efforts and Guarantees of Environmental Rights in Gunning Leuser National Park, Indonesia', JILS. Hein Online 2: 55

MacKinnon J, Phillipps K, Balen B. 2010. Birds in Sumatra, Java, Bali, and Kalimantan (Including Sabah, Sarawak and Brunei Darussalam), Burung Indonesia, Bogor, Indonesia. [indonesian]

Maia RC, Coutinho R. 2012. Structural characteristics of mangrove forests in Brazilian estuaries: A comparative study. Revista de Biología Marina y Oceanografía 47 (1): 87-98.

Morelli F. 2013. Quantifying effects of spatial heterogeneity of farmlands on bird species richness by means of similarity index pairwise, Intl $\mathrm{J}$ Biodiv 2013: 914837. DOI: 10.1155/2013/914837

Mundkur T, Langendoen T, Watkins D. 2017. The Asian Waterbird Census 2008-2015-Results of Coordinated Counts in Asia and Australasia, Wetlands International, Ede.

Nasution A. 2017. The government decentralization program in Indonesia, in Central and Local Government Relations in Asia. Edward Elgar Publishing, Cheltenham, UK.

Newbold T, Scharlemann JPW, Butchart SHM, Şekercioğlu CH, Alkemade R, Booth H, Purves DW. 2013. Ecological traits affect the response of tropical forest bird species to land-use intensity. Proc $\mathrm{R}$ Soc B Biol Sci 280 (1750): 20122131. DOI: 10.1098/rspb.2012.2131

Noerdjito M, Maryanto I. 2001. Jenis-Jenis Hayati yang Dilindungi Perundang-Undangan Indonesia. Puslitbang Biologi-LIPI, Bogor. [Indonesian]

Ong JE, Gong WK. 2013. Structure, function and management of mangrove ecosystems. International Society for Mangrove Ecosystems, Japan.

Perera K, Amarasinghe M. D, Somaratna S. 2013. Vegetation structure and species distribution of mangroves along a soil salinity gradient in a micro-tidal estuary on the north-western coast of Sri Lanka, Am J Mar Sci 1 (1): 7-15.

Raharjeng A, Noviyanti D, Apriana E, Fahriati L, Hastati YD. 2019. Plant diversity on protected forest vegetation of Dempo Mountains, Pagar Alam, South Sumatera. Jurnal Biota 5 (1): 42-47. [indonesian]
Rani V, Sreelekshmi S, Asha CV, Bijoy Nandan S. 2018. Forest structure and community composition of Cochin Mangroves, South-West Coast of India. Proc Natl Acad Sci India B Biol Sci 88 (1): 111-119.

Raymakers C. 2006. CITES, the Convention on International Trade in Endangered Species of Wild Fauna and Flora: its role in the conservation of Acipenseriformes. J Appl Ichthyol 22: 53-65.

Reeve R. 2014. Policing international trade in endangered species: the CITES treaty and compliance. Routledge, London.

Saenger: 2013. Mangrove ecology, silviculture and conservation. Springer Science \& Business Media, New York.

Sandilyan S, Kathiresan K. 2012. Mangrove conservation: a global perspective, Biodiv Conserv 21 (14): 3523-3542.

Şekerci A. D, Gülșen O. 2016. Overview of dahlia breeding, Scientific Papers-Series B Horticulture 60: 199-204.

Singh VP, Odaki K. 2004. Mangrove ecosystem: structure and function. Scientific Publishers, New Delhi.

Stirling G, Wilsey B. 2001. Empirical relationships between species richness, evenness, and proportional diversity, Amer Nat 158 (3): 286-299.

Sudrajat S, Putro MD. 2019. The contribution of forest remnants within industrial area to threatened mammal conservation: A case study in liquefied natural gas industry in Bontang, Indonesia. Biodiversitas 20: 2257-2265.

Sukardjo S. 1993. The present status of the mangrove forests in the northern coast of West Java with special reference to the recent utilization. Jpn J Southeast Asian Stud 31 (2): 141-157.

Tauseef M, Ihsan F, Nazir W, Farooq J. 2012. Weed flora and importance value index (IVI) of the weeds in cotton crop fields in the region of Khanewal, Pakistan. Pak J Weed Sci Res 18 (3): 319-330.

Tomlinson PB. 2016. The Botany of Mangroves. 2nd ed. Cambridge University Press, Cambridge, UK.

Widyastuti A, Yani E, Nasution EK, Rochmatino. 2018. Diversity of mangrove vegetation and carbon sink estimation of Segara Anakan Mangrove Forest, Cilacap, Central Java, Indonesia. Biodiversitas 19: 246-252. 